\title{
Nonlinear Optics on the Silicon Platform
}

\author{
W. Freude ${ }^{1,2}$, L. Alloatti ${ }^{1}$, A. Melikyan ${ }^{1}$, R. Palmer ${ }^{1}$, D. Korn ${ }^{1}$, N. Lindenmann ${ }^{1}$, T. Vallaitis ${ }^{1,3}$, D. Hillerkuss ${ }^{1}$, \\ J. Li ${ }^{1}$, A. Barklund ${ }^{4}$, R. Dinu ${ }^{4}$, J. Wieland ${ }^{4}$, M. Fournier ${ }^{5}$, J. Fedeli ${ }^{5}$, S. Walheim ${ }^{6,7}$, P. M. Leufke ${ }^{6}$, S. Ulrich $^{8}$,

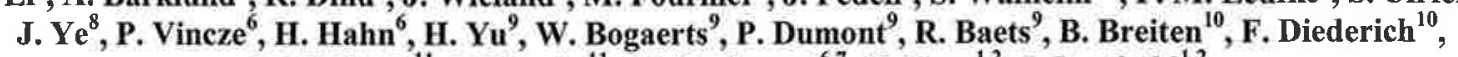 \\ M. T. Beels ${ }^{11}$, I. Biaggio ${ }^{11}$, Th. Schimmel ${ }^{6,7}$, C. Koos ${ }^{1,2}$, J. Leuthold ${ }^{1,2}$ \\ 'Institute of Photonics and Quantum Electronics (IPQ). Karlsruhe Institute of Technology (KIT), 76131 Karlsruhe, Germany \\ ${ }^{2}$ Institute of Microstructure Technology (IMT), Karlsruhe Institute of Technology (KIT), 76344 Eggenstein-Leopoldshafen, Germany \\ ${ }^{3}$ Now with: Infinera Corporation. 169 Java Drive, Sunnyvale, CA 94089, USA \\ ${ }^{4}$ GigOptix Inc, Switzerland and GigOptix Bothell, Washington, USA, ${ }^{5}$ CEA, LETI. Minatec 17 rue des Martyrs, 38054 Grenoble, France \\ ${ }^{6}$ Institute of Nanotechnology (INT), Karlsruhe Institute of Technology (KIT), 76344 Eggenstein-Leopoldshafen, Germany \\ ${ }^{7}$ Institute of Applied Physics, Karlsruhe Institute of Technology (KIT), 76131 Karlsruhe, Germany \\ ${ }^{8}$ Institute for Applied Materials (IAM-AWP), Karlsruhe Institute of Technology (KIT), 76344 Eggenstein-Leopoldshafen, Germany \\ ${ }^{9}$ Photon. Res. Group, Ghent Univ. - IMEC, Dpt. of' Informat. Technol. (INTEC) \& Center of Nano- and Biophoton. (NB-Photonics), Gent. Belgium \\ ${ }^{10}$ Laboratorium für Organische Chemie, ETH Zürich, 8093 Zürich, Switzerland, "Dpt. of Physics, Lehigh University, Bethlehem, PA 18015, USA \\ Email: w.freude@kit.edu
}

\begin{abstract}
Silicon represents a mature, affordable platform for fabricating electronic and optical signal processing devices. We discuss all-optical $170 \mathrm{Gbit} / \mathrm{s}$ switching, a $42 \mathrm{Gbit} / \mathrm{s}$ electro-optic modulator, and proof-of-concept results for a surface plasmon polariton absorption modulator. OCIS codes: (190.0190) Nonlinear optics, (130.0130) Integrated optics, (130.4815) Optical switching devices, 130.7405 Wavelength conversion devices, (130.4110) Modulators, 250.5403 Plasmonics
\end{abstract}

\section{Introduction}

Nonlinear effects in silicon support a large assortment of techniques for processing optical signals near wavelengths of $1.55 \mu \mathrm{m}$ at very high speed [1]. Especially the silicon-on-insulator (SOI) platform - typically a $220 \mathrm{~nm}$ thin silicon slab on top of a thick silicon oxide layer residing on a silicon substrate — allows strong field confinement in high index-contrast waveguides, thereby enhancing the native $\chi^{(3)}$-nonlinear response of silicon. The technology lends itself also to co-integrate electronic CMOS components [2], and to hybridly integrate active III-V [3] or Si/Ge devices [4]. The use of silicon nanocrystals [5] or amorphous silicon [6], and the addition of organic materials $[7,8,9]$ (silicon-organic hybrid, $\mathrm{SOH}$ ), of graphene $[10,11]$, or of metallic structures $[12,13]$ widens the scope even more by providing what silicon misses: A TPA-free $\chi^{(3)}$ and a $\chi^{(2)}$-nonlinearity. In the following, we choose from the diverse applications of silicon-based devices [14] three important examples that exploit nonlinear effects on the silicon platform: An all-optical switch, a high-speed electro-optic modulator, and a plasmonic absorption modulator.

\section{All-optical switching with FWM and XPM in $\chi^{(3)}$-nonlinear strip and SOH slot waveguides}

The SOI waveguides under consideration are depicted in Fig. 1a,b. The large $\chi^{(3)}$-nonlinearity of silicon cannot be fully exploited for fast all-optical switching due to the long lifetime and the loss of free carriers generated by twophoton absorption (TPA) [7,8]. To avoid these limitations experienced with a strip waveguide covered with air only, Fig. 1a(field lower left), we employ a highly nonlinear organic material $[7,15]$ that does not suffer from TPA and has ä low refractive index. Such SOH systems combine the strengths of both materials resulting in extremely large effective nonlinearities $[7,9,15,8]$. The SOH waveguides are silicon strips or vertical-slot SOI-structures (Fig. 1a,b) with a cladding of $\chi^{(3)}$-nonlinear organic material (DDMEBT [15]). The resulting SOH waveguide is described by a complex nonlinearity (NL) parameter $\gamma$. For maximum NL we need to optimize $\operatorname{Re}\{\gamma\}=n_{2} k_{0} / A^{(3)}$ eff (effective area $A^{(3)}$ eff, vacuum wave number $k_{0}$, nonlinear-index coefficient $\left.n_{2}\right)$. Optimized horizontal-slot quasi-TM waveguides were published recently [16]. TPA is quantified by a figure of merit $\mathrm{FOM}_{\mathrm{TPA}}=-\operatorname{Re}\left\{\gamma_{\}} /(4 \pi \operatorname{Im}\{\gamma\})\left(=n_{2} /\left(\alpha_{2} \lambda\right)\right.\right.$ with spatially homogeneous cross-section, TPA coefficient $\left.\alpha_{2}\right)$. For the structures in Fig. 1a,b we measured [8]: Linear loss $\alpha_{0 \text { strip }}=1 \mathrm{~dB} / \mathrm{mm}, \alpha_{0 \text { slot }}=1.5 \mathrm{~dB} / \mathrm{mm} ; \mathrm{FOM}_{\mathrm{TPA} \text { core }}=0.38, \mathrm{FOM}_{\mathrm{TPA} \text { clad }}=1.2, \mathrm{FOM}_{\mathrm{TPA} \text { slot }}=2.2 ; \operatorname{Re}\{\gamma\}_{\mathrm{NL}}$ in core $=307 /(\mathrm{Wm}), \operatorname{Re}\{\gamma\}_{\mathrm{NL} \text { in clad }}=108 /(\mathrm{Wm}), \operatorname{Re}\{\gamma\}_{\mathrm{NL} \text { in slot }}=100 /(\mathrm{Wm})$. For FOM $\mathrm{TPA}_{\mathrm{TPA}}>0.5$, TPA can be neglected.

We demonstrated the high-speed capability of nonlinear SOH slot waveguides with a number of experiments. Four-wave mixing (FWM) as in Fig. 2 demultiplexed a $170.8 \mathrm{Gbit} / \mathrm{s}$ OTDM signal to its four $42.7 \mathrm{Gbit} / \mathrm{s}$ tributaries [15]. The same setup is used for wavelength conversion with retiming. By FWM, a $42.7 \mathrm{Gbit} / \mathrm{s}$ RZ-OOK data signal at $1559 \mathrm{~nm}$ and a $42.7 \mathrm{GHz}$ clock at $1550 \mathrm{~nm}$ generate a converted signal at $1541 \mathrm{~nm}$ with a quality factor of $Q^{2}=11.3 \mathrm{~dB}$ and on-chip powers for data (clock) of $P_{1}=11.3 \mathrm{dBm}(21 \mathrm{dBm})$. We performed a similar experiment with NRZ DPSK data at $56 \mathrm{Gbit} / \mathrm{s}$ using an SOH strip waveguide as in Fig. 1a(field plot lower right). Finally, we demonstrated the transfer of $42.7 \mathrm{Gbit} / \mathrm{s} 33 \% \mathrm{RZ}$-OOK PRBS data at $1541 \mathrm{~nm}$ to a CW carrier at $1544.5 \mathrm{~nm}$ via cross-phase modulation (XPM). In all cases, no bit pattern dependence was to be seen. 

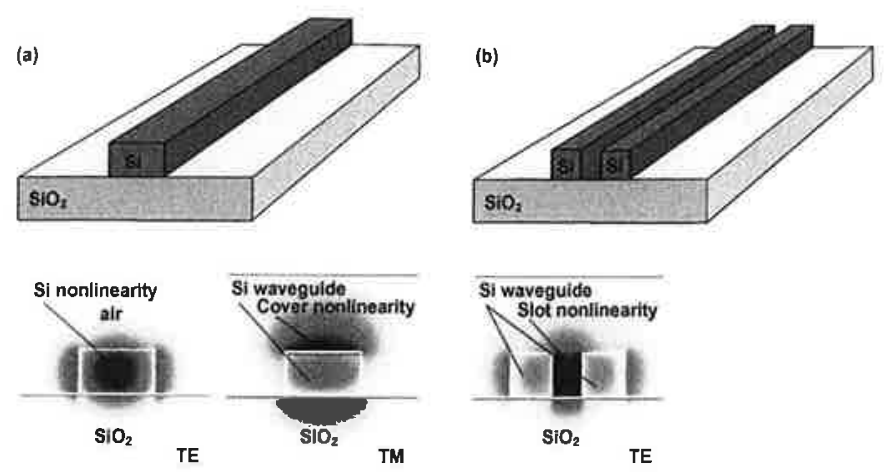

Fig. 1. Silicon waveguides (height $220 \mathrm{~nm}$ ) with air or organic cover and electric field magnitudes [8]. (a) Si strip, width $360 \ldots 400 \mathrm{~nm}$, quasi-TE mode, $\mathrm{SiO}_{2} /$ air cladding, Si core nonlinearity (lower left), and quasi-TM mode, strong cover nonlinearity (lower right). (b) Quasi-TE Si slot waveguide, rail widths $220 \mathrm{~nm}$, slot width $160 . .200 \mathrm{~nm}$, cladding/slot nonlinearity

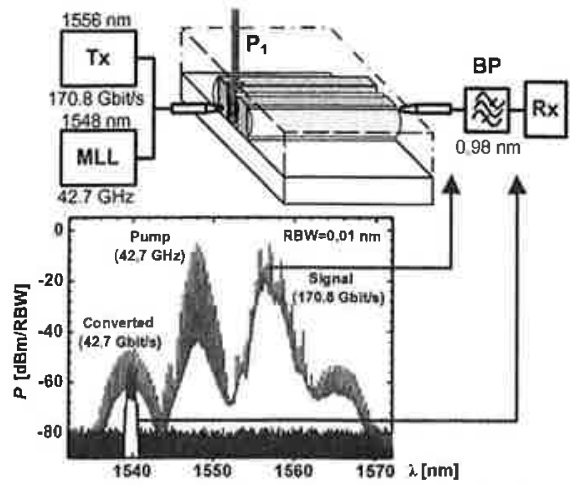

Fig. 2. Setup for FWM demultiplexer with $4 \mathrm{~mm}$ long SOH slot waveguide, Fig. 1b. Output spectrum before and after bandpass filter BP [15]. Tx: transmitter; Rx: receiver; MLL: mode-locked laser

\section{Electro-optic modulator with $\mathcal{X}^{(2)}$-nonlinear SOH slot waveguide}

The CMOS-compatible SOH approach for optical modulators exploits the properties of a $\chi^{(2)}$-nonlinear organic material which covers a slot waveguide, Fig.1b and Fig. 3a. A metallic travelling-wave transmission line connects the modulator voltage to the electro-optic active slot region. It must be both optically transparent and electrically highly conductive, so we induce a highly conductive electron accumulation layer by an external DC "gate" voltage $V_{\text {gate. }}$ As opposed to doping, the electron mobility in this case is not impaired by impurity scattering. Using a first-generation device at a data rate of $42.7 \mathrm{Gbit} / \mathrm{s}$, widely open eye diagrams were recorded [17], Fig. 4. The measured frequency response suggests that significantly larger data rates are feasible. Compared to a recently published similarly fast $p n-$ junction modulator [18], our device is more broadband $(>60 \mathrm{~nm})$ and more sensitive $\left(V_{\pi}=9 \mathrm{Vmm} @ 1 \mathrm{kHz}\right)$.

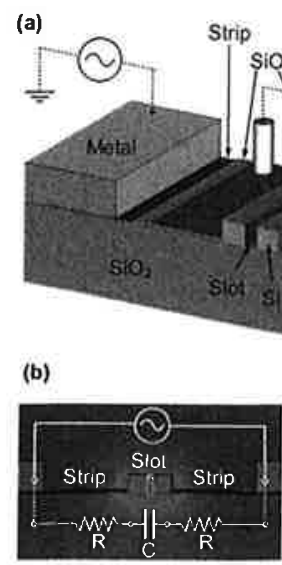
chromophores dispersed in amorphous polycarbonate, APC). Electro-optic coefficient with optimum in situ poling $r_{33}=70 \mathrm{pm} / \mathrm{V}$. (a) Silicon strips connect optical region with metal electrodes. A positive gate voltage $V_{\text {gate }}$ bends the bands $\left(E_{C V F}\right.$ : conduction, valence band, and Fermi energy; $q$ : elementary charge) resulting in a highly conductive electron accumulation layer. (b) Waveguide cross-section and electric optical field magnitude with equivalent circuit ( $C$ : slot capacitance; $R$ : strip resistance). [Reprint from [17] (C) 2011 OSA]

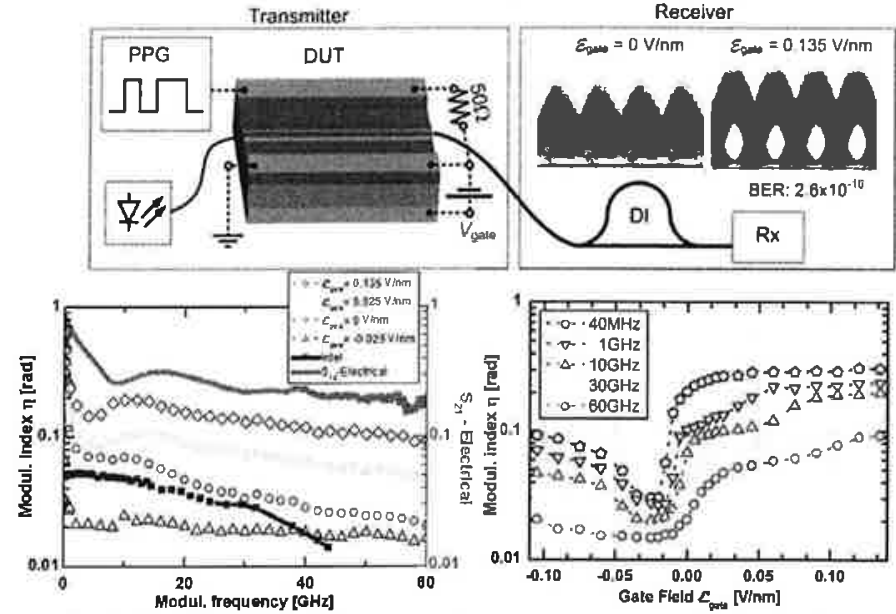

Fig. 4. SOH modulator (simplified realization compared to Fig.3a) and experimental results. (upper row) Setup with $42.7 \mathrm{Gbit} / \mathrm{s}$ pulse pattern generator (PPG) and delay interferometer (DI) for phase-to-amplitude conversion. (lower row) Phase modulation index $\eta$ for various gate electric field strengths $\varepsilon_{\text {pate }}$ and sinusoidal modulation voltages with $1 \mathrm{~V}$ amplitude and frequencies $f_{\text {mod }}=1 \mathrm{kHz} . .60 \mathrm{GHz}$. For large $\left|\varepsilon_{\text {gate }}\right|$ the SI strips become more conductive due to an electron accumulation (hole inversion) layer for $\varepsilon_{\text {alc }}>0.025 \mathrm{~V} / \mathrm{nm}\left(\varepsilon_{\text {gale }}<-0.025 \mathrm{~V} / \mathrm{nm}\right)$. Transmission of electrical waveguide (voltage ratio $\left|S_{21}\right|,-$ ). For $\varepsilon_{\text {gale }}=0.135 \mathrm{~V} / \mathrm{nm}$ and $f_{\text {mod }}=1 \mathrm{kHz}$ $(60 \mathrm{GHz})$ we found $V_{\pi} L=9 \mathrm{Vmm}(58 \mathrm{Vmm})$ corresponding to $V_{\pi}=5.3 \mathrm{~V}(34 \mathrm{~V})$. Flat response for $f_{\text {mod }}>2 \mathrm{GHz}$, suggesting that data rates could be extended well beyond the 42.7 Gbit/s limit of our equipment. [Reprinted from [17] (C 2011 OSA]

\section{Surface plasmon polariton absorption modulator}

To reduce the modulator footprint even further, an electrically controlled ultra-compact surface plasmon polariton (SPP) absorption modulator (SPPAM) was investigated. The device can be as short as $10 \mu \mathrm{m}$, depending on the re- 


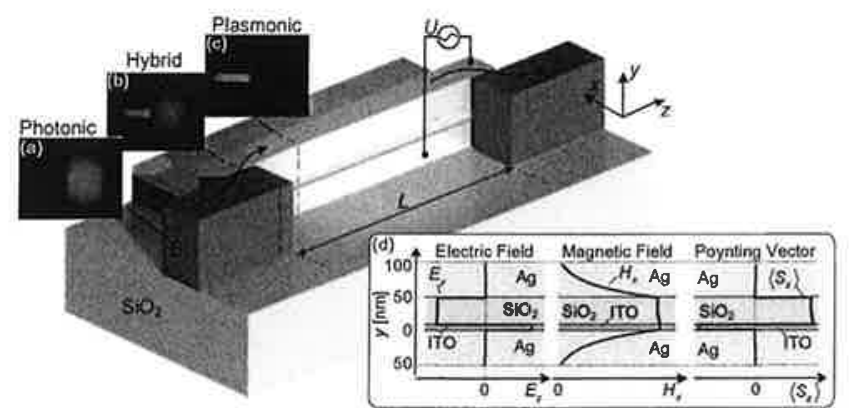

Fig. 5 Surface plasmon polariton (SPP) absorption modulator (SPPAM). With a directional coupler, light is coupled from a silicon nanowire into an active plasmonic section, consisting of stacked layers of silver $(\mathrm{Ag})$, indium tin oxide (TTO, $10 \mathrm{~nm}$ ), and $\mathrm{SiO}_{2}$. The SPP absorption coefficient is modulated by a voltage $U$ between the silver electrodes. Insets: The photonic mode (a) in the silicon nanowire excites via a hybrid mode (b) in the directional coupler an SPP (c). Inset (d) shows the electric field $E_{y}$, the magnetic field $H_{x}$, and the time-averaged Poynting vector $\left\langle S_{z}\right\rangle$ in the active plasmonic region, demonstrating the strong SPP confinement in the ITO layer. The modulator length is $L=10 \mu \mathrm{m}$. [Reprint from [19] (C 2011 OSA]

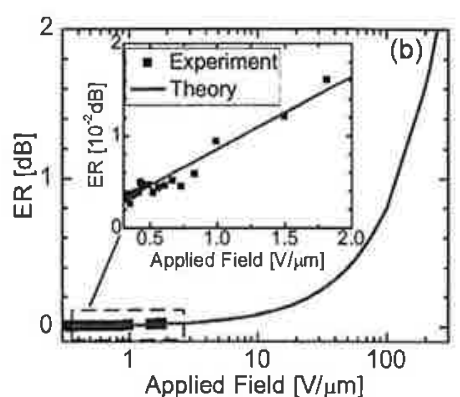

Fig. 6. Measured ( $\square$ ) and predicted (-) extinction ratio (ER) at $\mathrm{kHz}$-frequencies as a function of the applied modulation field. An ER of $1 \mathrm{~dB}$ is obtained with an electric field of $100 \mathrm{~V} / \mu \mathrm{m}$. Such a field strength can be easily achieved in the structure Fig. 5, and is far below the dielectric strength in the order of $10^{3} \mathrm{~V} / \mu \mathrm{m}$ for materials like $\mathrm{SiO}_{2}$ and $\mathrm{Si}_{3} \mathrm{~N}_{4}$. [Reprint from [19]@2011 OSA]

quired extinction ratio (ER) and the acceptable loss. The absorption modulator Fig. 5 comprises a stack of metal / insulator / metal-oxide / metal layers, which supports a strongly confined SPP in the $1.55 \mu \mathrm{m}$ wavelength region. The absorption is modulated by electrically changing the free carrier density in the intermediate metal-oxide layer. A three-layer prototype was designed, and the concept is supported by proof-of-principle experiments, Fig. 6.

Acknowledgements We acknowledge support by the DFG Center for Functional Nanostructures (CFN), the KIT Initiative of Excellence, the Karlsruhe School of Optics and Photonics (KSOP), EU-FP7 projects EURO-FOS (grant 224402), SOFI (grant 248609), EU-COST Action MP0702, and by the BMBF project MISTRAL (grant 01BL0804). We further acknowledge technological support by the Karlsruhe Nano-Micro Facility (KNMF), by the Light Technology Institute (KIT-LTI) and the ePIXfab (silicon photonics platform). W. B. acknowledges the Flemish Research Foundation (FWO-Vlaanderen) for a postdoctoral fellowship, I. B. partial support from the Commonwealth of Pennsylvania, Ben Franklin Technology Development Authority, and F. D. support from the ETH research council. We thank J. Groß and S. Schneider (KIT-IPQ) for help with the modulator design. We thank M. Pauli and K. P. Pahl (KIT-IHE), H. Kleinjans, M. Schmidt and M. Karl (AMO), and F. Milesi (CEA-LETI) for valuable discussions, and we are grateful to D. Gerthsen (KIT-LEM) for support in nano-inspection and analysis.

\section{References}

[1] J. Leuthold, C. Koos, and W. Freude, "Nonlinear silicon photonics," Nature Photon. 4, 535-544 (2010).

[2] W. M. J. Green, S. Assefa, A. Rylyakov, C. Schow, F. Horst, and Y. A. Vlasov, "CMOS integrated silicon nanophotonics: An enabling technology for exascale computing, "in OSA Proc. Conf. $\underline{\text { PR '11 }}$, June 12-16, 2011, Toronto, Canada. Paper IME1.

[3] M. J. R. Heck, H.-W. Chen, A. Fang, B. R. Koch, Di Liang, Hyundai Park, M. N. Sysak, and J. E. Bowers, "Hybrid silicon photonics for

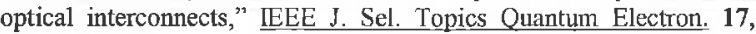
333-346 (2011).

[4] C. R. Doen, P. Winzer, Y.-K. Chen, S. Chandrasekhar, M. S. Rasras, Long Chen, T.-Y. Liow, K.-W. Ang, and G.-Q. Lo. "Polarization and phase diversity coherent receiver in silicon," J. Lightw. Technol. 28, 520-525 (2010).

[5] Z. Yuan, A. Anopchenko, N. Daldosso, R. Guider, D. NavarroUrios, A. Pitanti, R. Spano, and L. Pavesi, "Silicon nanocrystals as an enabling material for silicon photonics," Proc. IEEE 97, 1250-1268 (2009).

[6] K. Narayanan and S. F. Preble, "Optical nonlinearities in hydrogenated-amorphous silicon waveguides," Opt. Express 18, 8998-9005 (2010).

[7] C. Koos, L. Jacome, C. Poulton, J. Leuthold, and W. Freude, "Nonlinear silicon-on-insulator waveguides for all-optical signal processing," Opt. Express 15, 5976-5990 (2007).

[8] T. Vallaitis, S. Bogatscher, L. Alloatti, P. Dumon, R. Baets, M. L. Scimeca, I .Biaggio, F. Diederich, C., W. Freude, and J. Leuthold, "Optical properties of highly nonlinear silicon-organic hybrid (SOH) waveguide geometries," Opt. Express 17, 17357-17368 (2009).

[9] J. Leuthold, W. Freude, J.-M. Brosi, R. Baets, P. Dumon, I. Biaggio, M. L. Scimeca, F. Diederich, B. Frank, and C. Koos, "Siliconorganic hybrid (SOH) - A platform for ultrafast optics," IEEE Proc. 97, 1304-1316 (2007).
[10] A. K. Geim and K. S. Novoselov, "The rise of graphene," Nature Mat. 6, 183-191 (2007).

[11] M. Liu, X. Yin, E. Ulin-Avila, B. Geng, T. Zentgraf, L. Ju, F. Wang, and $\mathrm{X}$. Zhang, "A graphene-based broadband optical modulator," Nature 474,64-67 (2011).

[12] I. D. Rukhlenko, M. Premaratne, and G. P. Agrawal, "Nonlinear propagation in silicon-based plasmonic waveguides from the standpoint of applications," Opt. Express 19, 206-217 (2011).

[13] M. L. Brongersma and V. M. Shalaev, "The case for plasmonics," Science 328, 440-441 (2010).

[14] R, Soref, "Silicon photonics: A review of recent literature," Silicon 2, 1-6 (2010)

[15] C. Koos, P. Vorreau, T. Vallaitis, P. Dumon, W. Bogaerts, R. Baets, B. Esembeson, I. Biaggio, T. Michinobu, F. Diederich, W. Freude, and J. Leuthold, "All-optical high-speed signal processing with silicon-organic hybrid slot waveguides," Nature Photon, 3, 216219 (2009)

[16] P. Muellner, M. Wellenzohn, and R. Hainberger, "Nonlinearity of optimized silicon photonic slot waveguides," Opt. Express 17, 9282-9287 (2009.

[17] L. Alloatti, D. Kom, R. Palmer, D. Hillerkuss, J. Li, A. Barklund, R. Dinu, J. Wieland, M. Foumier, J. Fedeli, H. Yu, W. Bogaerts, P. Dumon, R. Baets, C. Koos, W. Freude, and J. Leuthold, "42.7 Gbit/s electro-optic modulator in silicon technology," Opt. Express 19, 11841-11851 (2011).

[18] D. J. Thomson, F. Y, Gardes, Y. Hu, G. Mashanovich, M. Fournier, P. Grosse, J.-M. Fedeli, and G. T. Reed, "High contrast 40 Gbit/s optical modulation in silicon," Opt. Express 19, 11507-11516 (2011). [19] A. Melikyan, N. Lindenmann, S. Walheim, P. M. Leufke, S. Ulrich, J. Ye, P. Vincze, H. Hahn, Th. Schimmel, C. Koos, W. Freude, and J. Leuthold, "Surface plasmon polariton absorption modulator," Opt.Express 19, 8855-8869 (2011). 